\title{
Accelerating MEMS Development by Open Collaboration at Hands-On-Access Fab, Tohoku University
}

\author{
Kentaro Totsu, Masaaki Moriyama, Yukio Suzuki, and Masayoshi Esashi \\ Micro System Integration Center, Tohoku University \\ 519-1176 Aramaki-Aza-Aoba, Aoba-ku, Sendai 980-0845, Japan
}

(Received November 10, 2017; accepted December 26, 2017)

Keywords: open facility, sharing, accumulated know-how, training program, commercialization

The hands-on-access fabrication facility for MEMS at Tohoku University is an open facility for every engineer working to develop MEMS devices. The fab is located at Junichi Nishizawa Memorial Research Center, Tohoku University, and was started in 2010. The operation of the fab is based on open collaboration wherein all persons involved in MEMS development share the facilities, human resources, technology, and know-how to overcome the difficulties in MEMS development. The users of the fab can utilize the fab and operate the equipment by themselves with the support of the technical staff. The users also can access accumulated know-how at Tohoku University. Know-how, in particular, the conditions and results of each fabrication process, is shared by the users and the staff. Over 200 companies have utilized the fab for developing various devices, such as an accelerometer, pressure sensor, photodiode, radiation sensor, solar cell, microphone, and micromirror. There have already been several commercialized MEMS devices that have been fully or partly developed at the fab. Simultaneous nurturing of engineers and the development of MEMS devices through on-thejob training at the fab effectively contributes to device commercialization. To accelerate the university's research, development, and education, product fabrication by a company user was started in July 2013.

\section{Introduction}

MEMS development requires specific resources including facilities, know-how, and engineers, because MEMS is versatile and hence difficult to standardize. The facility includes equipment such as those for photolithography, deposition, etching, and testing, and cleanroom environments. Know-how includes technology of the total MEMS development process, which consists of device and system design, fabrication process, and testing. Engineers should be experienced in such total MEMS development processes. Preparing and maintaining MEMS resources in-house consumes much time and cost. Therefore, a company planning to develop MEMS devices for the first time tends to collaborate with an institution that can offer MEMS resources. Such a collaborating institution includes universities, institutes, MEMS foundries,

*Corresponding author: e-mail: totsu@tohoku.ac.jp

http://dx.doi.org/10.18494/SAM.2018.1804 
and companies dedicated to contract development. As for human resources, if an engineer who has experience with MEMS in the company that is going to start development, joins the MEMS development as the counterpart of the collaborator, the development could go forward. On the other hand, the joint development could be in a difficult situation if only inexperienced engineers join the counterpart. In particular, misunderstanding often occurs when the development proceeds only on the collaborator side by contract. In our experience with collaborations among many companies, having an experienced MEMS engineer on the company side is the key to successful MEMS development. Therefore, if there are no appropriate MEMS engineers in the company, the company should hire an experienced MEMS engineer, or train such an engineer. Experienced companies that have already developed MEMS resources inhouse often look for collaborators to make up for deficiencies in technologies, facilities, and nurturing of MEMS engineers. In this background, we started an open access facility, named "Hands-On-Access Fabrication Facility for MEMS at Tohoku University" in 2010, where any engineer can utilize MEMS facilities and know-how. The fab was statred as part of the project "Integrated Microsystems", conducted under the direction by Professor Masayoshi Esashi at Tohoku University, the main researcher of one of the Japanese Funding Programs for WorldLeading Innovative R\&D on Science and Technology (FIRST). The project was concluded in March 2014. Another national program for open facilities, "Nanotechnology Platform" of the Ministry of Education, Culture, Sports, Science and Technology (MEXT), has supported the Hands-On-Access fab since 2012. The fab activity was supported by the Creation of Innovation Centers for Advanced Interdisciplinary Research Areas Program, MEXT, until 2017.

The fab is equipped mainly with facilities for 4- and 6-inch MEMS and semiconductor development. To fabricate prototype MEMS and semiconductor devices, a company can access the fab and operate the equipment themselves. Since standardization of the MEMS process is quite difficult, MEMS engineers are required to choose appropriate processes from among numerous candidates. This process design approach requires much know-how, which is not easily transferred from one person to another. The hands-on system contributes to the development of human resources having practical experience, which is strongly required for device development. The fab does not accommodate contract development like a foundry, however, the fab offers not only technical operational support but also know-how accumulated at the university over 40 years. The fab is shared with other users. Therefore, sharing the costs of the installation and maintenance of the fab can reduce the risk in device development.

Company users pay fees to the university in accordance with their usage time and equipment. Users can reduce their investment cost, because they can fabricate prototypes of a device at reasonable cost. As a result, users can reduce the risk in development, and can transition from the $R \& D$ stage to the production stage more smoothly.

Since the 1970s, Tohoku University has provided an open access lab where company engineers have worked to develop MEMS devices, supported by university staff. The lab offers a $20 \times 20 \mathrm{~mm}^{2}$ wafer processing facility. The flexibility at the facility that allows users to modify machines to serve their research needs is a great advantage in accelerating $R \& D$ work, because it enables users to efficiently confirm the principle of a new device in development and its fabrication process, particularly in the early stage of the research effort. In order to increase 
the probability of successful commercialization, we needed 4- and 6-inch wafer process facilities that can provide equipment directly connected to commercialization to company users. Company users can fine-tune their devices and repeat prototyping to fit their application in the market.

\section{Structure of the Fab}

\subsection{Facility}

Hands-on-access fabrication is carried out at Jun-ichi Nishizawa Memorial Research Center, Tohoku University, in Sendai, Japan (Fig. 1). The research center was a part of the Semiconductor Research Institute. In 2008, the foundation was dissolved and all properties of the foundation were donated to Tohoku University. The research center is a three-story building, of which the first, second, and parts of the third floor are cleanrooms. Each floor has a $1800 \mathrm{~m}^{2}$ area. Hands-on-access fabrication is mainly carried out on the second floor and occupies about $1200 \mathrm{~m}^{2}$ of floorspace (Fig. 2). In that area, there was a 4-inch-wafer process facility that had been utilized for manufacturing power transistors by Tokin and its successor company. The 4-inch-wafer facility also became part of Tohoku University, because the company ceased their operation in 2008. Fortunately, we did not need to install new equipment, but merely restarted the old equipment. Although it was originally a 4-inch-wafer facility, we modified the facility for 6-inch wafers. Additionally, we installed some new equipment to realize the MEMS process as well as the semiconductor process in 2010, supported by public funds. The fab has accepted over 20 donated secondhand pieces of equipment from companies. The total number of pieces of equipment at the fab in 2017 exceeded 120. This equipment can be applied to mainly the following processes: cleaning, drying, etching, CVD, photolithography, oxidation, diffusion, ion implantation, sputtering, evaporation, bonding, packaging, imprinting, and evaluation. $^{(1)}$

In the research center, the Sendai MEMS Show Room is located on the second floor. ${ }^{(2)}$ The show room is managed by the MEMS Park Consortium. The content includes MEMS devices that have been developed at the university and other institutions. Visitor can get a feel of the history of MEMS development in the world, and in particular, understand the flow of the commercialization of MEMS.

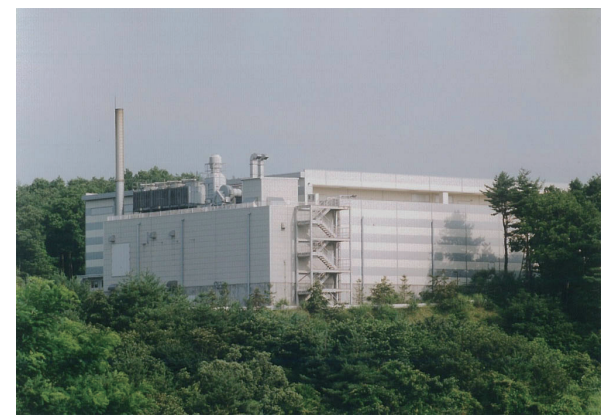

Fig. 1. (Color online) Jun-ichi Nishizawa Memorial Research Center, Tohoku University. 


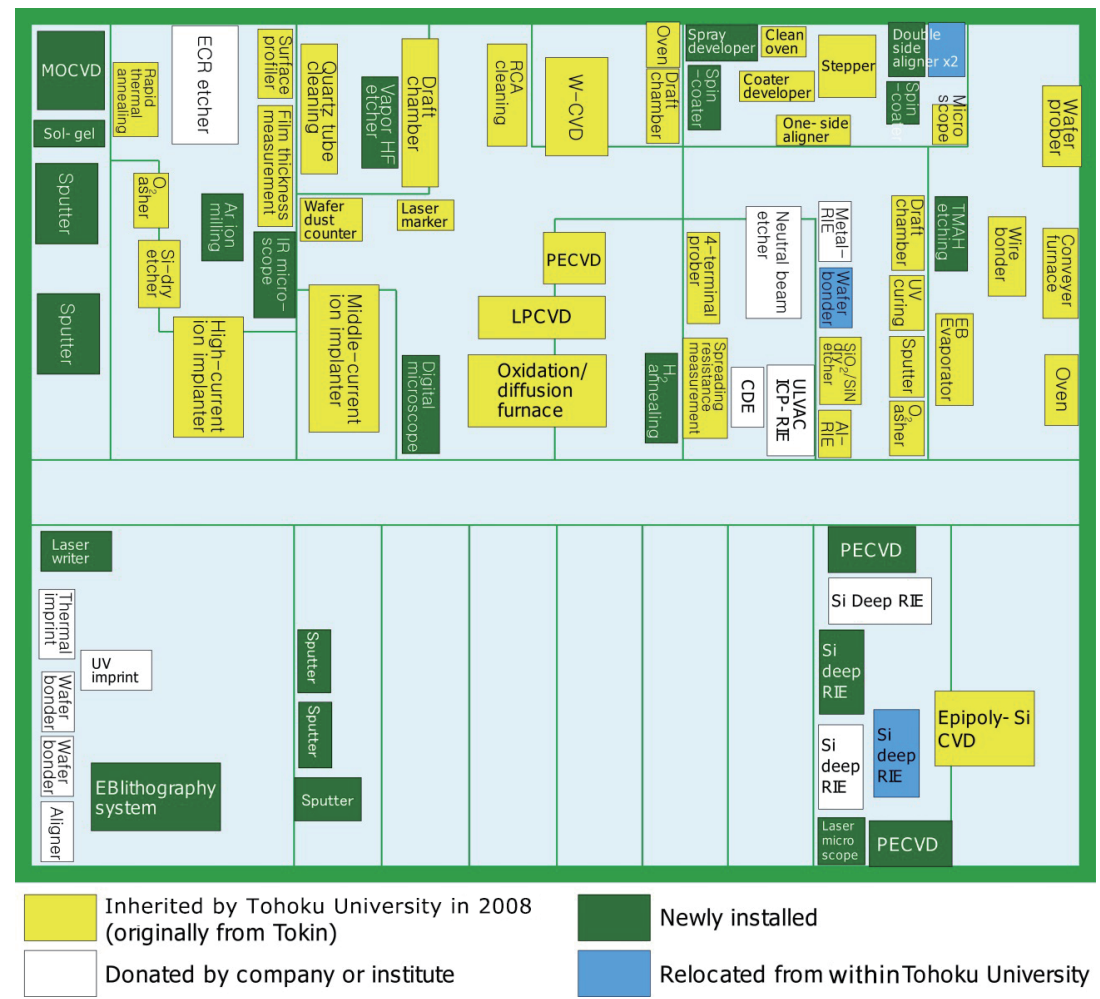

Fig. 2. (Color online) Layout of clean room for hands-on access fab.

\subsection{Support staff}

Nine engineering support staff members (three research and six technical staff members) are working together at the hands-on-access fab. All members have MEMS and/or semiconductor backgrounds. Four of the technical staff members worked at the Semiconductor Research Institute, which was the former manifestation of the research center, and have knowledge of and practical experience with silicon and compound semiconductor process, as well as the whole research center facility. Two technical staff members worked with the company that manufactured power transistors at the same facility until 2008. Although the main task of the staff is to support company users, they also maintain the equipment and develop fundamental processes.

\subsection{Technology}

The hands-on-access fab can offer standard process recipes to users, on the basis of accumulated know-how at the university. If a user needs to develop an original process recipe, the fab staff supports the user as much as they can.

Process development is an important activity at the fab. Low-stress CVD, deposition of piezoelectric material for actuators, and various etching processes are examples of achievement.

A deposition technique of epitaxial poly-Si with low stress, a high-speed deposition rate, 
and high conductivity has been realized. This process has been successfully applied to the fabrication of an optical scanner without the conventional silicon on insulator (SOI) wafer process. ${ }^{(3)}$

A stress-controlled fabrication process has been applied for a subwavelength grid infrared cut filter as large as $90 \mathrm{~mm}$ in diameter. ${ }^{(4)}$ A large free-standing submicron honeycomb structure has been successfully fabricated by carefully balancing film stress at each process step.

The fabrication of 300- $\mu \mathrm{m}$-deep through silicon vias (TSVs) in a CMOS-LSI wafer has been possible on a commercial multiproject wafer (MPW). ${ }^{(5)}$ The CMOS-LSI wafer and a MEMS wafer are integrated by $\mathrm{Au}-\mathrm{Au}$ thermocompression bonding at $300^{\circ} \mathrm{C}$. This fabrication process yields cost-effective surface-mountable CMOS-integrated MEMS.

As one MEMS packaging technology, a hermetic sealing method utilizing $\mathrm{Au}-\mathrm{Au}$ thermocompression bonding at $160{ }^{\circ} \mathrm{C}$ with a $\mathrm{Au}$ particle has been developed and is now available at MEMS Core, ${ }^{(6)}$ a company located in Sendai, that supports contract MEMS development and small-volume production. The compliance with bond surface irregularity as well as the insensitivity to surface flatness, thanks to the deformability of the Au particle, is advantageous to this hermetic sealing.

\subsection{Revenue}

Company users pay a fee in accordance with their usage. The fee in FY2017 consists of the following.

- Facility usage fee: JPY $880 / \mathrm{h}$

- Technical assistant fee: JPY 5565/h

- Equipment usage fee: JPY 1000-16000/h

- Material cost (chemicals, Au/Pt sputtering target, etc.)

If a company uses the hands-on-access fab for one week, the average payment is JPY 100000-200000. At the end of each month, the university calculates the fee for one month of usage for each company. Then in the middle of the next month, the university sends a bill to each company. If a company can disclose some results of the fabrication process, discounts on the technical assistant fee are applied through the Nanotechnology Platform program. The revenue and the expenditure of the hands-on-access fab in FY2016 are shown in Fig. 3. The annual revenue is JPY $182 \mathrm{M}$. Users fees amount to $71 \%$ of the revenue, while public funds obtained through the Nanotechnology Platform program make up 23\%.

\subsection{Training program}

In order to develop a MEMS research and industrial hub in Sendai, the MEMS Park Consortium was founded in 2004 with the strong support of Tohoku University, the City of Sendai, the Miyagi Prefectural Government, the Tohoku Bureau of Economy, Trade and Industry, the Development Bank of Japan, Tohoku Branch, the Tohoku Economic Federation, and SEMI Japan. One of the major activities of the consortium is organizing the training programs carried out at the hands-on-access fab. The original program was developed in 2006 


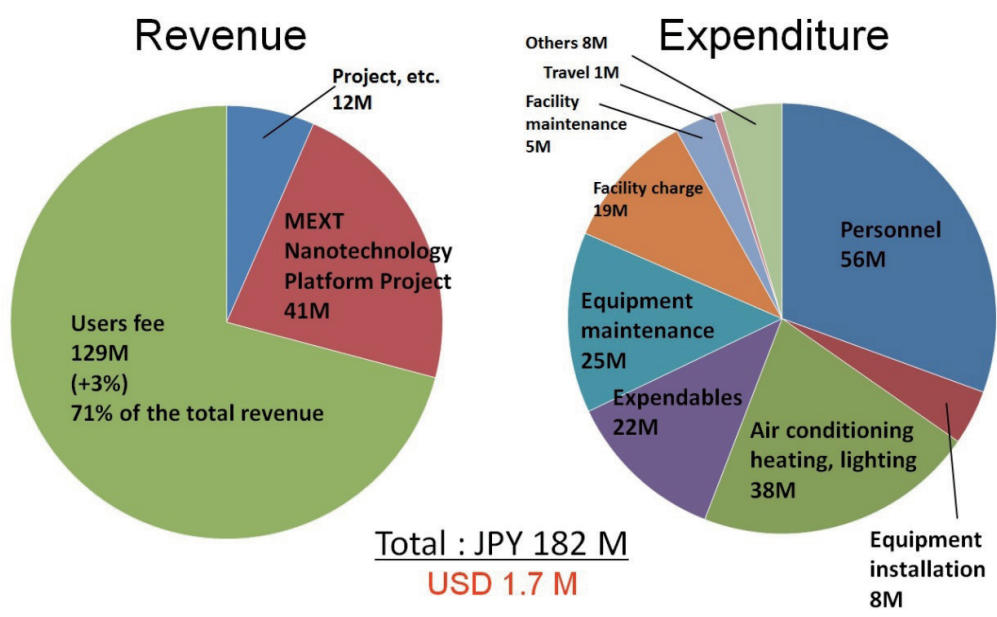

Fig. 3. (Color online) Revenue and expenditure in FY2016.

supported by the Ministry of Economy, Trade and Industry (METI). The program consists of basic lectures, planning and design, fabrication, testing and presentation, and report writing. The program takes three months and accepts a maximum of two or three engineers from different companies. The feature of this training program is that trainees can learn practical MEMS design and the fabrication process using their own devices. Every trainee can decide what to design, fabricate, and test in the three months of the program. The fabrication process is carried out by each trainee. Hence, trainees can learn the comprehensive process of MEMS development. This approach is very effective for mastering technical issues and knowhow. From 2013 to 2017, a MEMS training program supported by the City of Sendai was also provided. Every year, around five engineers having no experience with the MEMS process took part in the one-week program to create prototype MEMS devices, such as a pressure sensor, a force sensor, an accelerometer, and a mechanical switch on a SOI wafer (Fig. 4).

\section{Users}

\subsection{User list}

The total number of users for each month is shown in Fig. 5. The total number reaches 800 users per month. Over 200 companies have utilized the fab since it began full operations in November 2010. Names of company users that can be disclosed are shown in Fig. 6. The list includes not only device manufacturers but also material suppliers and process equipment manufacturers. Most of them repeatedly use the fab. The listed companies are mostly domestic, but the hands-on-access fab very much welcomes foreign companies.

Some companies are present at the fab almost constantly. Some companies are fabless, which means they have no facility for MEMS device development and production. In the 


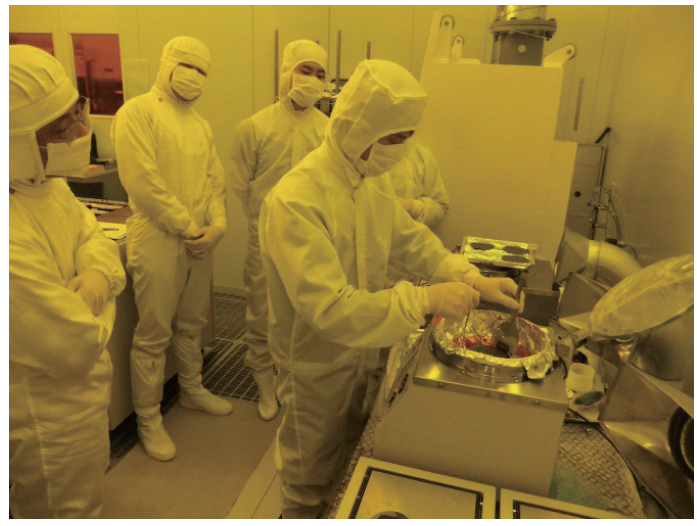

Fig. 4. (Color online) Hands-on MEMS training program.

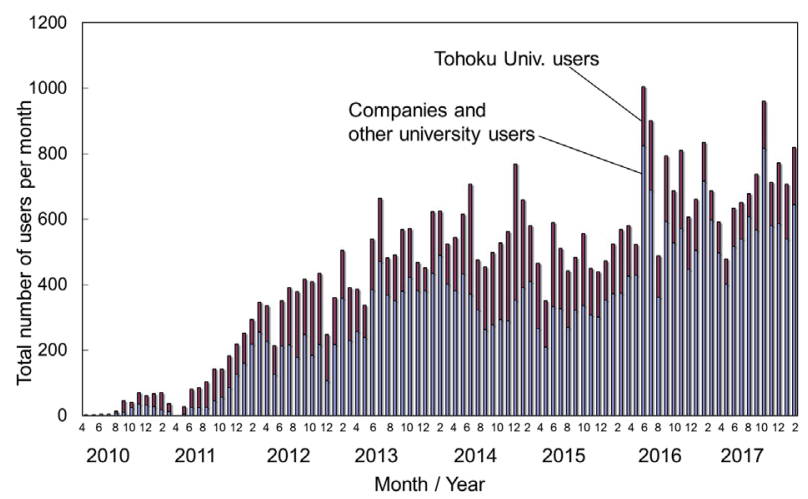

Fig. 5. (Color online) Total number of users every month.

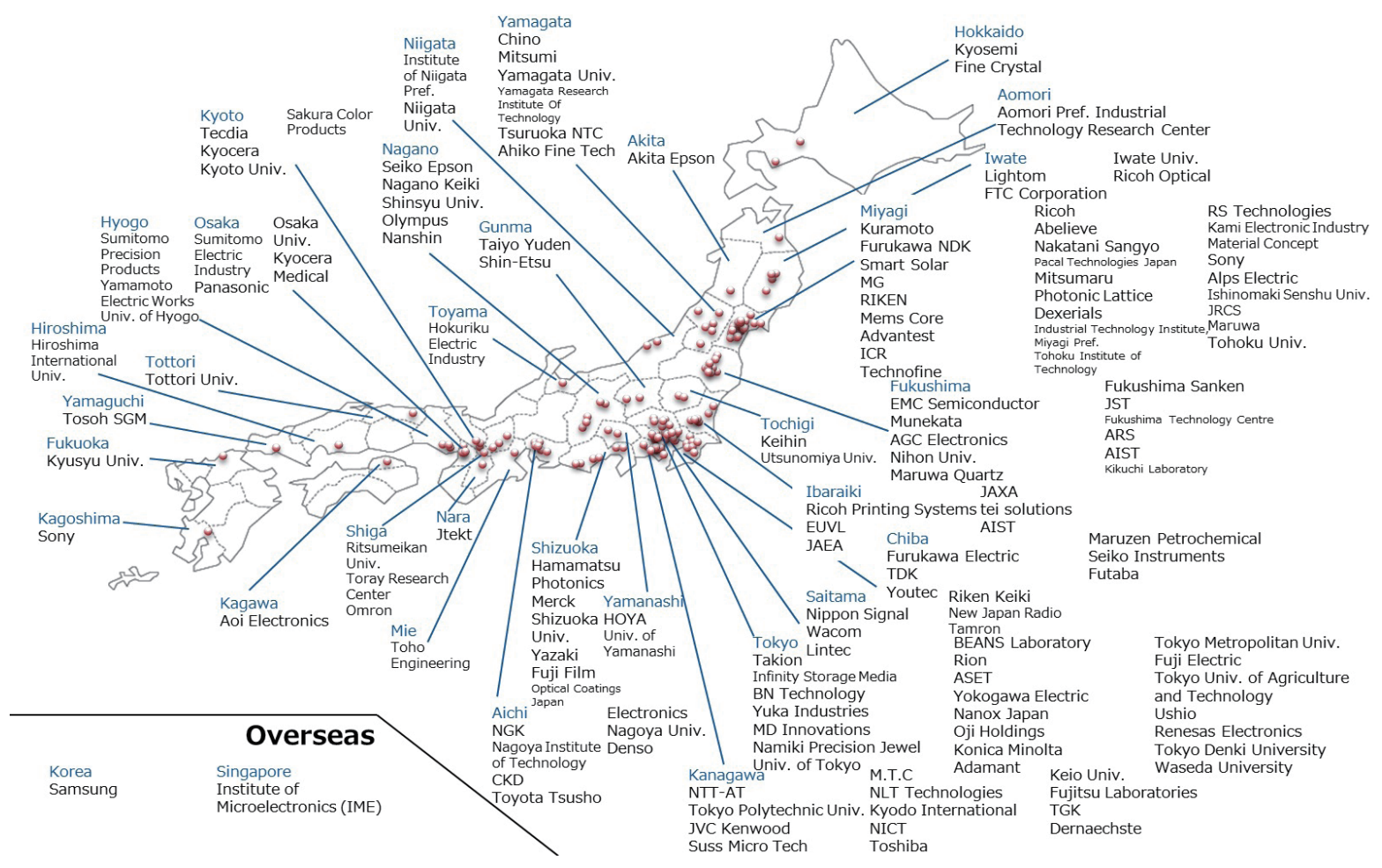

Fig. 6. (Color online) User list of hands-on-access fab since 2010.

MEMS industry, fabless models seem to be effective in reducing business risk. Some start-ups working on MEMS and micro-nano technology also utilize the fab. The fab strongly supports such fabless companies. 


\subsection{Developed and commercialized MEMS devices}

In the past, users have successfully developed photodiodes, magnetic sensors, pressure sensors, and microphones, ${ }^{(7)}$ for instance. A radiation sensor was commercialized just after the 2011 Great East Japan Earthquake .

The precise differential capacitive pressure sensor shown in Fig. 7 was commercialized by Yamamoto Electric Works in 2017. The pressure range of the most sensitive type reaches $50 \mathrm{~Pa}$. They started development in 2010 at the hands-on-access fab, through the three-month training program organized by the MEMS Park Consortium. We advised that they attempt to develop the sensor and nurture an engineer simultaneously, because they had never worked on MEMS before. After the successful fabrication of the first prototype in the training program, they continued their development at MEMS Core. In parallel, Yamamoto Electric Works developed and installed a facility for sensor packaging and testing in-house. At present, MEMS pressure chips continue to be fabricated at MEMS Core, and the sensor modules are manufactured and tested at Yamamoto Electric Works. The sensors have already been built into the process equipment for semiconductors.

A broadly tunable quantum cascade laser utilizing a MEMS micromirror, as shown in Fig. 8, was successfully demonstrated by Hamamatsu Photonics in 2017. Upon rotational vibration of the grating on the micromirror actuated by electromagnetic force, the cavity length of the laser changes. ${ }^{(8)}$ As a result, the wave number of the laser changes in the $200 \mathrm{~cm}^{-1}$ range with $9.0 \mu \mathrm{m}$ as the center wavelength. For instance, this broadly tunable infrared laser can be utilized for fast environmental gas monitoring. One reason why the company used the hands-on-access fab is that the engineer dedicated to this development had never worked with MEMS, even though the company has a history in MEMS and sufficient facilities for developing optical MEMS devices.

\section{Product Fabrication}

Product fabrication by company users has been possible at the hands-on-access fab since June 2013. The purpose of this product fabrication is to demonstrate the university's R\&D achievements in the form of a product in the market and society, and to obtain feedback

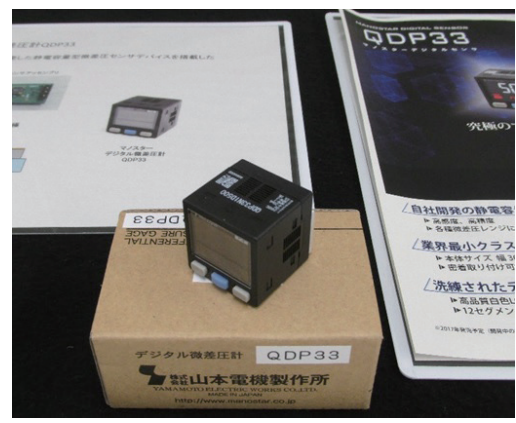

Fig. 7. (Color online) Precise differential pressure sensor module (30 $\mathrm{mm}$ cube), Yamamoto Electric Works. 


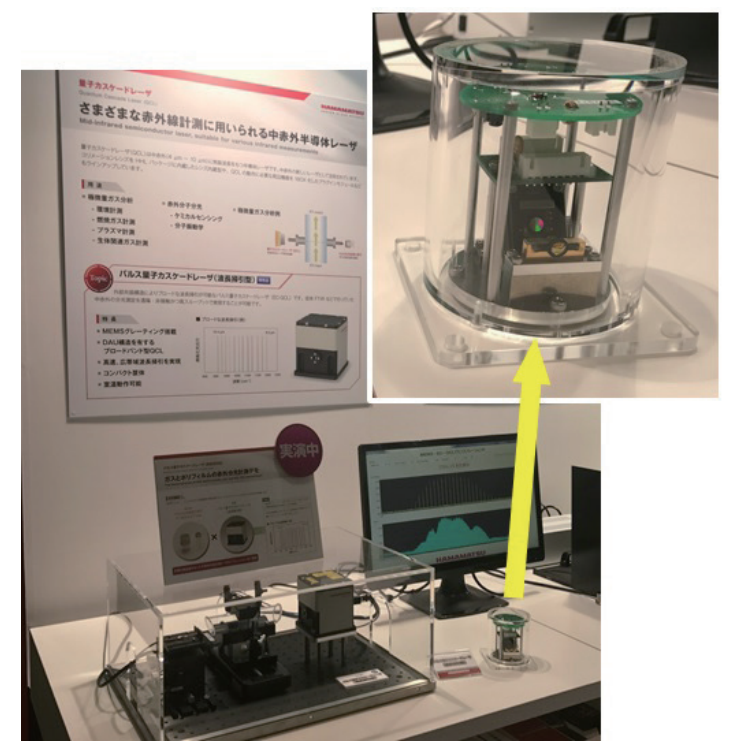

Fig. 8. (Color online) Broadly tunable quantum cascade laser, Hamamatsu Photonics, demonstrated at InterOpt in Tokyo, Oct. 2017.

regarding the results or any problems through production. We expect that this approach will accelerate the university's R\&D and education.

Product fabrication is carried out under the following conditions.

- The device should be developed with the cooperation of the university, and should be continuously developed at the fab.

- Occupied area and time should be less than $5 \%$ of the total capacity of the fab.

- A report to the university must be made every six months.

- Maximum period for one type of device product fabrication is three years.

- The university is exempt from responsibility.

Some companies have been carrying out product fabrication since 2013.

\section{Discussion}

The hands-on-access fab has been aiming to continuously support MEMS development and to establish a sustainable model of an open facility. The fab is now regarded as an almost established facility. On the basis of the experiences and the results described above, the key factors of its success are as follows.

(a) Facility

- Large-scale facility including 4" semiconductor fab, clean rooms, and donated equipment is effectively reutilized. The combination of very old and relatively new equipment provides flexibly to cover a wide range of MEMS development.

(b) Technology, know-how

- Users can access accumulated know-how at the university, as well as the latest information disseminated by the university. 
- Know-how, in particular, the conditions and results of each fabrication process, is shared with other users and staff.

(c) Human resources and its development

- Skillful staff members continuously and sincerely support users. The facility is mostly maintained by the staff.

- In principle, contract development is not accepted. Company engineers utilize the fab by themselves with the staff's support. This approach contributes to the development of engineers who gain sufficient knowledge through practical MEMS experience.

- Development of devices and nurturing of engineers are simultaneously carried out. This enables smooth technology transfer and has great potential for continuous collaborative $\mathrm{R} \& \mathrm{D}$ at the fab.

(d) Finance

- The Japanese government and local governments effectively support the fab.

(e) Network

- Seamless handling, from early development to production, has been realized in the Sendai area.

(f) University

- The university can gain valuable information regarding the demands of MEMS R\&D through their support of users. This effect renders R\&D at the university more active and practical.

- The fab has played an important role in technical incubation for start-ups.

The key factors above are based on open collaboration wherein all the persons involved in MEMS development and support share facilities, human resources, and know-how to overcome the difficulties in MEMS development.

\section{Conclusions}

An open facility named the hands-on-access fab at Tohoku University has been utilized by more than 200 companies since 2010. Several MEMS devices have been commercialized by the company users. In particular, the simultaneous nurturing of engineers and development of MEMS devices through on-the-job training at the fab effectively contributes to device commercialization. The fab is now being successfully operated on the basis of the concept of open collaboration, wherein MEMS engineers from companies and the university share facilities, human resource, and know-how. The fab is expected to be a sustainable platform for accelerating MEMS development, as well as a place where engineers and technology congregate.

\section{Acknowledgments}

Hands-on-access fab was supported by the Japanese Funding Programs for World-Leading Innovative R\&D on Science and Technology (FIRST), JSPS, the Creation of Innovation Centers for Advanced Interdisciplinary Research Areas Program, MEXT, and METI. The fab is now supported by the Nanotechnology Platform, MEXT. 


\section{References}

1 http://www.mu-sic.tohoku.ac.jp/coin_e/index.html.

2 http://www.mu-sic.tohoku.ac.jp/showroom_e/index.html.

3 Y. Suzuki, K. Totsu, H. Watanabe, M. Moriyama, M. Esashi, and S. Tanaka: IEEJ Trans. SM 133 (2013) 223.

4 Y. Suzuki, K. Totsu, M. Moriyama, M. Esashi, and S. Tanaka: Sens. Actuators, A 231 (2015) 59.

5 Y. Suzuki, H. Fukushi, M. Muroyama, Y. Hata, T. Nakayama, R. Chand, H. Hirano, Y. Nonomura, H. Funabashi, and S. Tanaka: Proc. IEEE MEMS 2017, Las Vegas, USA (2017) 744.

6 T. Ogashiwa, K. Totsu, M. Nishizawa, H. Ishida, K. Inoue, Y. Sasaki, M. Miyairi, S. Tanaka, and M. Esashi: Proc. Transducers 2017, Kaohsiung, Taiwan (2017) 1304.

7 Y. Fuji, M. Hara, Y. Higashi, S. Kaji, K. Masunishi, T. Nagata, A. Yuzawa, K. Otsu, K. Okamoto, S. Baba, T. Ono, A. Hori, and H. Fukuzawa: Proc. Transducers 2017, Kaohsiung, Taiwan (2017) 63.

8 A. Sugiyama, N. Akikusa, and T. Edamura: Proc. 34th Sensor Symposium, Hiroshima, Japan (2017) 31am3PS-27. 